\title{
Asymptotically exact solution of the multi-channel resonant-level model
}

\author{
Guang-Ming Zhang*, Zhao-Bin Su田, and Lu Yut \\ International Center for Theoretical Physics, P. O. Box 586, 34100 Trieste, Italy
}

\begin{abstract}
An asymptotically exact partition function of the multi-channel resonant-level model is obtained through Tomonaga-Luttinger bosonization. A Fermi-liquid vs non-Fermi-liquid transition, resulting from a competition between the Kondo and X-ray edge physics, is elucidated explicitly via the renormalization group theory. In the strong-coupling limit, the model is renormalized to the Toulouse limit.
\end{abstract}

PACS Numbers: $72.10 . \mathrm{Bg}, 71.28 .+\mathrm{d}$ 
The central issue under current debate is whether one should involve non-Fermi liquid (FL) behavior to interpret the normal state properties of the high- $T_{c}$ superconductors and whether such a behavior can be derived from models of strong electron correlations [1,2]. Except for one dimension, perturbation treatments can not provide any mechanism for the breakdown of FL behavior [3]. Thus, it is of great interest to study models whose properties can be obtained either exactly or within controllable approximations, exhibiting a transition from FL to non-FL behavior as some parameters of the models are varied.

Unfortunately, lattice models have so far proven intractable to show such a transition. On the other hand, some single-impurity models, for example, single-channel Anderson or Kondo model, show a local FL behavior [4], whereas the others, e.g., the X-ray edge [5] or the multi-channel Kondo problem [6,7] exhibit non-FL characteristics. Recently, Varma et al. have explored the possible FL-non-FL transitions in the generalized Anderson model [8] and its spinless version: multi-channel resonant-level (MCRL) model $[9,10]$, which should shed some light on similar transitions in lattice models. Another type of generalized Anderson model has been also considered in the context of infinite dimensions [11].

The single-channel resonant-level model can be derived from the Kondo model through Tomonaga-Luttinger bosonization and it exhibits the same local FL behavior below the Kondo temperature as for the Anderson impurity model $[12,13]$. The generalization of this model to include finite range Coulomb repulsion leads to a coupling to more orbital channels in addition to the hybridizing channel itself [9]. The spinless version of this generalized Anderson impurity model may contain the basic physics of the FL vs non-FL transition in the symmetric case [8].

In this Rapid Communication, we first use bosonization [14] to derive the asymptotically exact partition function for the MCRL model. Then, we apply the poor-man's scaling theory $[15]$ to calculate the renormalization group $(R G)$ equations from which the physics of a FL-non-FL transition is explicitly demonstrated. In fact, we can separate the model Hamiltonian into "hybridizing" and "screening" parts. The first part is just the usual singlechannel resonant-level model describing the Kondo physics, exhibiting a local FL behavior 
$[12,13]$, while the second part is the multi-channel X-ray edge model describing the Anderson orthogonality catastrophe [5], displaying a local non-FL behavior. The essential physics of the MCRL model is thus the competition between the FL-type Kondo resonance and the non-FL-type Anderson catastrophe. When the screening part has at least three channels and strong enough repulsive interactions with impurity, the model displays the X-ray edge singularities; otherwise, the model is describing the Kondo physics of the hybridizing part. There exists a transition between these FL and non-FL metallic phases, mathematically similar but physically different from the usual Kosterlitz-Thouless phase transition corresponding to a metal-insulator transition [16]. The transition concerned here is just a crossover behavior rather than a genuine phase transition.

The MCRL model Hamiltonian is given by [10]

$$
H=\sum_{k, l} \epsilon_{k} C_{k, l}^{\dagger} C_{k, l}+\frac{t}{\sqrt{N}} \sum_{k}\left(C_{k, 0}^{\dagger} d+d^{\dagger} C_{k, 0}\right)+\frac{1}{N} \sum_{k, k^{\prime}, l} V_{l} C_{k, l}^{\dagger} C_{k^{\prime}, l}\left(d^{\dagger} d-\frac{1}{2}\right)
$$

where $C_{k, l}\left(C_{k, l}^{\dagger}\right)$ are spinless conduction electrons with orbital momentum or channel index $l$, and $d\left(d^{\dagger}\right)$ operators correspond to a localized impurity. As required by symmetry, the localized impurity hybridizes only with one-channel, $l=0$. The chemical potential is set to zero, and the model has particle-hole symmetry. It should be noted that this model Hamiltonian is exactly the spinless version of the generalized Anderson impurity model [8].

In order to reveal the physics contained in this model, we first separate the model Hamiltonian into two part, $H=H_{h}+H_{s}$, where

$$
\begin{gathered}
H_{h}=\sum_{k} \epsilon_{k} C_{k, 0}^{\dagger} C_{k, 0}+\frac{t}{\sqrt{N}} \sum_{k}\left(C_{k, 0}^{\dagger} d+d^{\dagger} C_{k, 0}\right)+\frac{V_{0}}{N} \sum_{k, k^{\prime}} C_{k, 0}^{\dagger} C_{k^{\prime}, 0}\left(d^{\dagger} d-\frac{1}{2}\right), \\
H_{s}=\sum_{k, l \neq 0} \epsilon_{k} C_{k, l}^{\dagger} C_{k, l}+\frac{1}{N} \sum_{k, k^{\prime}, l \neq 0} V_{l} C_{k, l}^{\dagger} C_{k^{\prime}, l}\left(d^{\dagger} d-\frac{1}{2}\right) .
\end{gathered}
$$

The hybridizing part $H_{h}$ is just the single-channel resoant-level model, corresponding to the single-channel Kondo problem [12,13], while the screening part $H_{s}$ is the multi-channel X-ray edge problem [5]. When these two parts are coupled through the impurity, there appears an interesting competition between these two different kinds of metallic behavior. 
First, we deal with $H_{s}$ by bosonization [14]. If only s-wave scattering is concerned, this model can be reduced to a one-dimensional problem with one Fermi point for each screening channel. We introduce the density operators

$$
\rho_{l}(k)=\frac{1}{\sqrt{N}} \sum_{q=0}^{k_{D}-k} C_{q, l}^{\dagger} C_{q+k, l}, \quad \rho_{l}(-k)=\frac{1}{\sqrt{N}} \sum_{q=k}^{k_{D}} C_{q, l}^{\dagger} C_{q-k, l}, \quad k \geq 0,
$$

and consider a band of width $k_{D}$. The electron energies are given by $\epsilon_{k}=\left(k-k_{F}\right) / \rho$, where $\rho \equiv\left(h v_{F}\right)^{-1}$ is the density of states at the Fermi point. We can define bosonic operators $b_{k, l}=\frac{1}{\sqrt{k}} \rho_{l}(k), b_{k, l}^{\dagger}=\frac{1}{\sqrt{k}} \rho_{l}(-k)$, which obey the standard commutation relations. Thus, the screening part $H_{s}$ can be transformed into

$$
H_{s}=\sum_{k, l>0} \frac{k}{\rho} b_{k, l}^{\dagger} b_{k, l}+\sum_{k, l>0} \frac{V_{l}}{\sqrt{N}} \sqrt{k}\left(b_{k, l}+b_{k, l}^{\dagger}\right)\left(d^{\dagger} d-\frac{1}{2}\right)
$$

and it can be further diagonalized to $\sum_{k, l>0} \frac{k}{\rho} b_{k, l}^{\dagger} b_{k, l}$ through the canonical transformation

$$
U=\exp \left\{\sum_{k, l>0} \frac{\rho V_{l}}{\sqrt{k N}}\left(b_{k, l}-b_{k, l}^{\dagger}\right)\left(d^{\dagger} d-\frac{1}{2}\right)\right\}
$$

In the mean time, the above transformation also transfers the singularities of the screening channels into the hybridizing part via the localized impurity. The model Hamiltonian thus becomes

$$
H=\sum_{k} \epsilon_{k} C_{k}^{\dagger} C_{k}+\frac{1}{\sqrt{N}} \sum_{k}\left(\Delta^{\dagger} C_{k}^{\dagger} d+\Delta d^{\dagger} C_{k}\right)+\frac{V_{0}}{N} \sum_{k, k^{\prime}} C_{k}^{\dagger} C_{k^{\prime}}\left(d^{\dagger} d-\frac{1}{2}\right)+\sum_{k, l>0} \frac{k}{\rho} b_{k, l}^{\dagger} b_{k, l}
$$

with $\Delta \equiv t e^{\left\{-\sum_{k, l>0} \frac{\rho V_{l}}{\sqrt{k N}}\left(b_{k, l}-b_{k, l}^{\dagger}\right)\right\}}$. Here we have omitted the index "0" for the hybridizing channel. This is a modified single channel resonant-level model with an effective hybridization strength $\Delta$, which can change the behavior of the model from FL with canonical exponents to non-FL with singular nonuniversal exponents. This resulting Hamiltonian is exact and includes all physical features of the MCRL model.

Next, we derive the partition function of the Hamiltonian (5). As in the Kondo problem $[17,18]$, this Hamiltonian is divided as: $H=H_{0}+H_{I}$,

$$
H_{0}=\sum_{k} \epsilon_{k} C_{k}^{\dagger} C_{k}+\frac{V_{0}}{N} \sum_{k, k^{\prime}} C_{k}^{\dagger} C_{k^{\prime}}\left(d^{\dagger} d-\frac{1}{2}\right)+\sum_{k, l>0} \frac{k}{\rho} b_{k, l}^{\dagger} b_{k, l}
$$




$$
H_{I}=\frac{1}{\sqrt{N}} \sum_{k}\left(\Delta^{\dagger} C_{k}^{\dagger} d+\Delta d^{\dagger} C_{k}\right)
$$

$H_{0}$ describes a two-state system where the impurity is either empty or filled, while $H_{I}$ plays the role of a dipole operator causing transitions between these two states. The spinless fermion opators $d, d^{\dagger}$ can be exactly mapped onto Pauli matrices as $\sigma^{z}=2 d^{\dagger} d-1, \sigma^{+}=d^{\dagger}$, $\sigma^{-}=d$. For the partition function $Z$ in the interaction representation (with $\beta$ as the inverse temperature), we have $Z=\operatorname{Tr}\left(e^{-\beta H_{0}} T e^{-\int_{0}^{\beta} H_{I}(\tau) d \tau}\right)$, where $H_{I}(\tau)=e^{H_{0} \tau} H_{I} e^{-H_{0} \tau}$ and $T$ is the time ordering operator. The partition function can be expanded as: $Z=\sum_{n} Z_{n}$. We introduce the following notations in the presence (absence) of the impurity:

$$
H_{0}^{ \pm}=\sum_{k} \epsilon_{k} C_{k}^{\dagger} C_{k} \pm \frac{V_{0}}{2 N} \sum_{k, k^{\prime}} C_{k}^{\dagger} C_{k^{\prime}}+\sum_{k, l>0} \frac{k}{\rho} b_{k, l}^{\dagger} b_{k, l},
$$

and $C_{0}=\frac{1}{\sqrt{N}} \sum_{k} C_{k}$. When performing tracing over impurity's configurations, we find that $Z_{n}$ vanishes for odd $n$, while for even $n$ we obtain $Z_{n}=Z_{n}^{\prime}+Z_{n}^{\prime \prime}$ with

$$
Z_{n}^{\prime}=\int_{0}^{\beta} d \tau_{n} \int_{0}^{\tau_{n}-\tau_{0}} d \tau_{n-1} \ldots \int_{0}^{\tau_{2}-\tau_{0}} d \tau_{1}\left\langle e^{-\left(\beta-\tau_{n}\right) H_{0}^{+}}\left(\Delta C_{0}\right) e^{-\left(\tau_{n}-\tau_{n-1}\right) H_{0}^{-}}\left(\Delta^{\dagger} C_{0}^{\dagger}\right) \ldots\left(\Delta^{\dagger} C_{0}^{\dagger}\right) e^{-\tau_{1} H_{0}^{+}}\right\rangle .
$$

The expression for $Z_{n}^{\prime \prime}$ is obtained by interchanging $H_{0}^{+} \leftrightarrow H_{0}^{-}, \Delta^{\dagger} C_{0}^{\dagger} \leftrightarrow \Delta C_{0}$. Because of the rotational invariance, we have $Z_{n}^{\prime}=Z_{n}^{\prime \prime}$.

As for the screening channels, the bosonization can be used for hybridizing electrons as well, so far only s-wave scattering is involved. The boson operators are defined as

$$
a_{k}=\frac{1}{\sqrt{k N}} \sum_{q=0}^{k_{D}-k} C_{q}^{\dagger} C_{q+k}, \quad a_{k}^{\dagger}=\frac{1}{\sqrt{k N}} \sum_{q=k}^{k_{D}} C_{q}^{\dagger} C_{q-k}, \quad k \geq 0 .
$$

Restricted to the low-lying excitations, the kinetic energy can also be linearized to yield

$$
H_{0}^{ \pm}=\sum_{k>0} \frac{k}{\rho} a_{k}^{\dagger} a_{k} \pm \frac{V_{o}}{2 \sqrt{N}} \sum_{k>0} \sqrt{k}\left(a_{k}+a_{k}^{\dagger}\right)+\sum_{k, l>0} \frac{k}{\rho} b_{k, l}^{\dagger} b_{k, l},
$$

and $C_{0}=\left(\sqrt{k_{D}}\right) \exp \left\{\sum_{k>0} \frac{1}{\sqrt{k N}}\left(a_{k}-a_{k}^{\dagger}\right)\right\} . H_{0}^{ \pm}$describes two systems of harmonic oscillators with shifted origins. There is a similar canonical transformation $S=\exp \left\{\frac{\rho V_{0}}{2} \sum_{k>0} \frac{1}{\sqrt{k N}}\left(a_{k}-\right.\right.$ $\left.\left.a_{k}^{\dagger}\right)\right\}$ which connects the shifted oscillators to the unshifted ones. We then have $H_{0}^{+}=$ $S H_{f} S^{-1}, H_{0}^{-}=S^{-1} H_{f} S$, with $H_{f}=\sum_{k>0} \frac{k}{\rho} a_{k}^{\dagger} a_{k}+\sum_{k, l>0} \frac{k}{\rho} b_{k, l}^{\dagger} b_{k, l}$. Hence 


$$
Z_{n}^{\prime}=\int_{0}^{\beta} d \tau_{n} \int_{0}^{\tau_{n}-\tau_{0}} d \tau_{n-1} \ldots \ldots \int_{0}^{\tau_{2}-\tau_{0}} d \tau_{1}\left\langle B\left(i \tau_{n}\right) B^{\dagger}\left(i \tau_{n-1}\right) B\left(i \tau_{n-2}\right) \ldots \ldots B^{\dagger}\left(i \tau_{1}\right)\right\rangle
$$

where $B(t)=e^{i H_{f} t}\left(S^{-1} \Delta C_{0} S^{-1}\right) e^{-i H_{f} t} \equiv\left(t \sqrt{k_{D}}\right) e^{A(t)}$ and

$$
A(t)=\left(1-\rho V_{0}\right) \sum_{k>0} \frac{1}{\sqrt{k N}}\left(a_{k} e^{i \frac{k}{\rho} t}-a_{k}^{\dagger} e^{-i \frac{k}{\rho} t}\right)+\sum_{k, l>0} \frac{\rho V_{l}}{\sqrt{k N}}\left(b_{k, l} e^{i \frac{k}{\rho} t}-b_{k, l}^{\dagger} e^{-i \frac{k}{\rho} t}\right) .
$$

Now, we evaluate the thermodynamic expectation values using the strategies of Schotte [18] to obtain the partition function as

$$
Z=2 \sum_{n=0}^{\infty}(\tilde{t})^{2 n} \int_{0}^{\beta} \frac{d \tau_{n}}{\tau_{0}} \int_{0}^{\tau_{n}-\tau_{0}} \frac{d \tau_{n-1}}{\tau_{0}} \ldots . . \int_{0}^{\tau_{2}-\tau_{0}} \frac{d \tau_{1}}{\tau_{0}} \exp \left\{(1+\gamma) \sum_{i>j}(-1)^{i+j} \ln \left|\frac{\tau_{i}-\tau_{j}}{\tau_{0}}\right|\right\},
$$

where the bare dimensionless hybridizing strength $\tilde{t} \equiv \frac{\rho t}{\sqrt{k_{D}}}$, the cutoff factor $\tau_{0}=\frac{\rho}{k_{D}}$, and $\gamma \equiv-\frac{2 \delta_{0}}{\pi}+\sum_{l}\left(\frac{\delta_{l}}{\pi}\right)^{2} . \delta_{l}=\pi \rho V_{l}$ are the phase shifts in the Born approximation whose exact values should be $\delta_{l}=2 \tan ^{-1}\left(\frac{\pi}{2} \rho V_{l}\right)$. However, for weak interactions $V_{l}$ they are the same. Moreover, the total phase shift of the model is $\delta \equiv \frac{\pi}{2}(1+\sqrt{1+\gamma})[12]$. The form of the partition function we obtained is the same as that found by Anderson and Yuval for the Kondo problem [17]. Comparing our expression and theirs, we identify $\tilde{t} \leftrightarrow \rho J_{\perp} / 2$ and $(1+\gamma) \leftrightarrow\left(2-2 \rho J_{z}\right) .(1+\gamma)$ is the interaction strength between the spin-flips.

We also note that the above partition function for $\gamma=0$ is exactly the partition function of the following Hamiltonian:

$$
H_{T}=\sum_{k} \epsilon_{k} C_{k}^{\dagger} C_{k}+\frac{t}{\sqrt{N}} \sum_{k}\left(C_{k}^{\dagger} d+d^{\dagger} C_{k}\right)
$$

This is just the Toulouse limit [19] of the MCRL model, which is exactly the strong-coupling fixed point of this model, displaying a local FL behavior. When the model is renormalized to this fixed point, its physics can be described by a single-channel hybridization effect.

To set up the RG flow equations of the model, we can employ the poor-man's scaling procedure of Anderson et al. [15] in the Coulomb gas representation. The RG equations describe the flow of the hybridizing strength $\tilde{t}$ as the bandwidth $\frac{1}{\tau}$ is reduced. They are given by

$$
\begin{aligned}
& \frac{d \gamma}{d(\ln \tau)}=-4(1+\gamma)(\tilde{t})^{2}+O\left(\tilde{t}^{4}\right) \\
& \frac{d \tilde{t}}{d(\ln \tau)}=\frac{(1-\gamma)}{2} \tilde{t}+O\left(\tilde{t}^{3}\right)
\end{aligned}
$$


and $(\gamma-1)^{2}-16(\tilde{t})^{2}=$ const. These equations were derived by assuming a small fugacity, $\tilde{t} \ll 1$, i.e., a rare gas of spin-flips. In general, the average spacing between flips is much greater than $\tau$, i.e., $\tau \ll\left|\tau_{i}-\tau_{j}\right|$. As in the Kondo problem, we have ignored the explicit $\tau_{0}$ dependence of $\tilde{t}$. Our RG flow equations lead to two different types of behavior:

(i). For $\gamma<1$, or $\tilde{t} \geq \frac{1}{4}(\gamma-1)$ but $\gamma>1$, the hybridizing strength $\tilde{t}$ will always be relevant. The system is controlled by the strong-coupling fixed point, displaying FL-type universal asymptotic behavior. This fixed point is outside the range of validity of the poorman's RG approach, but it gives the correct flow direction. As seen from the partition function, when $\gamma=0$ the system will reach the unitary limit with the total phase shift $\delta=\pi$, which is nothing but exactly the Toulouse limit. Therefore, we can identify the strong-coupling fixed point with this limit.

We notice that the poor-man's scaling part of this model is similar to the single-channel Kondo problem, but the behavior in the strong-coupling regime is somehow different. Due to the emergence of new relevant variables, the Toulouse limit description for the strongcoupling limit of the Kondo problem is only qualitatively correct [20].

(ii). For $\gamma>1$ and $\tilde{t}<\frac{1}{4}(\gamma-1)$, the hybridization strength $\tilde{t}$ will be renormalized to zero, being an irrelevant variable even with repulsive interaction $V_{l}$. There exists a weakcoupling fixed line $\tilde{t}=0$. The system exhibits a power-law decay of correlation functions with a non-universal exponent, i.e., the same as for the multi-channel X-ray edge problem. Due to the large number of the screening channels, the orthogonality catastrophe effect is now sufficiently strong to drive the hybridization to zero. As seen from the expression for $\gamma$, at least three channels of conduction electrons with maximum phase shifts $\pi$ is needed to observe this effect. In this regime, the poor-man's scaling can be justified, so the results obtained are reliable and self-consistent.

The flow diagram is shown in Fig.1. When the bare hybridizing strength sits at a particular line, $\tilde{t}=\frac{1}{4}(\gamma-1)$, the system is in a marginal state: in the short-time (highenergy) limit, we obtain a non-FL singular scaling exponents, while in the long-time (low- 
energy) limit, a FL behavior will be observed. The system exhibits a nontrivial crossover between the FL and non-FL exponents. This means $\tilde{t}=\frac{1}{4}(\gamma-1)$ corresponds to the FLnon-FL separation line. Hence, we have given a complete physical picture of the FL-non-FL transition in the MCRL model, and have found that this model truly includes the basic physics of the generalized Anderson impurity model in the symmetric case [8].

From the above asymptotically exact solution of the MCRL model, we can see that the change of physics at $\gamma=1$ pointed out in Ref.[10] is basically correct. However, instead of studying the MCRL model itself, these authors considered an anisotropic multi-channel Kondo-like model, where the physics of competing X-ray edge singularities and Kondo behavior is not transparent. There is a crucial difference between the MCRL model and the multi-channel Kondo model. We would like to emphasize that the non-FL behavior in the MCRL model is caused by the X-ray edge singularity appearing in the weak coupling regime, while the physics of the two (or more) channel Kondo problem is the exhibition of a non-FL behavior in the strong coupling regime even after discarding the Anderson orthogonality catastrophe [7]. The origins of these two types of non-FL behavior might be different.

In conclusion, we have found an asymptotically exact solution of the MCRL model, and have given an explicit physical picture of the FL-non-FL crossover in this model.

Acknowledgments. G. M. Zhang is indebted to X. Y. Zhang for his many useful discussion and a earlier collaboration on the related subject. This work was supported in part by the Chinese Science Foundation for Young Scientists through Grant No. 19204010. 


\section{REFERENCES}

* Permanent address: Pohl Institute of Solid State Physics, Tongji University, Shanghai 200092, P. R. China.

$\dagger$ Permanent address: Institute of Theoretical Physics, Academia Sinica, P. O. Box 2735, P. R. China.

[1] . W. Anderson, Science 235, 1196 (1987); P. W. Anderson, Physica C 185-189, 11 (1991).

[2] . M. Varma, P. B. Littlewood, S. Schmitt-Rink, E. Abraham, and A. E. Ruckenstein, Phys. Rev. Lett. 63, 1996 (1989); A. E. Ruckenstein and C. M. Varma, Physica C 185-189, $134(1991)$.

[3] . W. Anderson, Phys. Rev. Lett. 71, 1220 (1993); R. Shankar, to be published in Revs. Mod. Phys. (1993).

[4] ee, e. g., P. Coleman, in Current Topics in Condensed Matter and Particle Physics, eds. J. Pati, Q. Shafi, and L. Yu, (World Sci. 1993).

[5] . W. Anderson, Phys. Rev. 164, 352 (1967); G. D. Mahan, ibid. 163, 612 (1967); P. Nozières and C. De Dominicis, ibid. 178, 1097 (1969); K. D. Schotte and U. Schotte, Phys. Rev. 182, 479 (1969).

[6] . Nozières and A. Blandin, J. Phys. (Paris), 41, 193 (1980); A. W. W. Ludwig and I. Affleck, Phys. Rev. Lett. 67, 3160 (1991).

[7] . J. Emery and S. Kivelson, Phys. Rev. B 46, 10812 (1992).

[8] . Perakis, C. M. Varma, A. E. Ruckenstein, Phys. Rev. Lett. 70, 3467 (1993).

[9] . M. Varma and T. Giamarchi, in Strongly Interacting Fermions and High Temperature Superconductivity, Proc. of the Les Houches Summer School, 1991, edited by B. Doucot (North-Holland, Amsterdam, 1992). 
[10] . Giamarchi, C. M. Varma, A. E. Ruckenstein, and P. Nozières, Phys. Rev. Lett. 70, 3967 (1993).

[11] . Si and G. Kotliar, Phys. Rev. Lett. 70, 3143 (1993).

[12] P. B. Wiegmann and A. M. Finkel'stein, Sov. Phys. JETP 48, 102 (1978).

[13] . Schlottmann, J. Phys. (Paris) C6, 1486 (1978); Phys. Rev. B 25, 4815 (1982).

[14] . Tomonaga, Progr. Theor. Phys. 5, 544 (1950); J. M. Luttinger, J. Math. Phys. 4, 1154 (1963); J. Sólyom, Adv. Phys. 28, 209 (1980).

[15]. W. Anderson, G. Yuval, and D. R. Hamann, Phys. Rev. B 1, 4464 (1970); P. W. Anderson, J. Phys. C 3, 2436 (1970).

[16] . M. Kosterlitz and D. J. Thouless, J. Phys. C 6, 1181 (1973); J. M. Kosterlitz, ibid. 7, 1046 (1974).

[17] . W. Anderson and G. Yuval, Phys. Rev. Lett. 23, 89 (1969).

[18] . D. Schotte, Z. Phys. 230, 99 (1970).

[19] . Toulouse, C. R. Acad. Sci. Paris, B 268, 1200 (1969).

[20] . Nozières, Proc. 15th Conf. Low Temp. Phys. Vol. 5, edited by M. Krusius and M. Vuorio, (Amsterdam, North-Holland), p. 339. 


\section{Figure Caption}

Fig.1 The RG flow diagram for the multi-channel resonant-level model derived from the scaling theory of Anderson et al. 


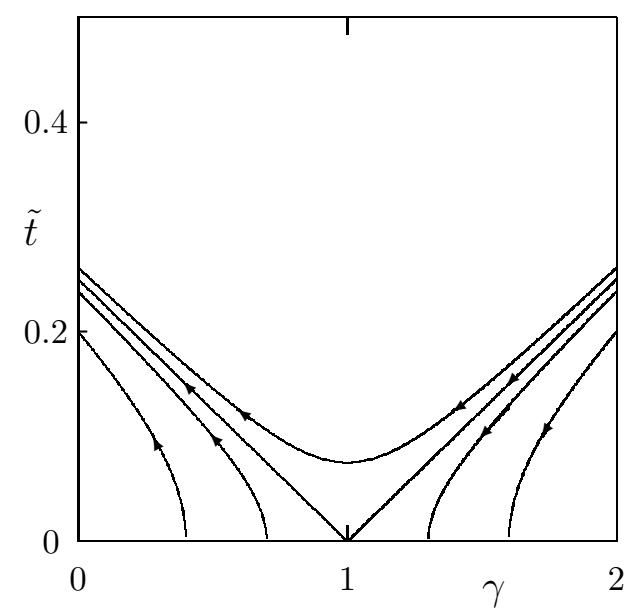

